\title{
Antagonistic implications of sarcopenia and abdominal obesity on physical performance in COPD
}

\author{
Coby van de Bool ${ }^{1}$, Erica P.A. Rutten², Frits M.E. Franssen², Emiel F.M. Wouters ${ }^{1,2}$ \\ and Annemie M.W.J. Schols ${ }^{1}$
}

\author{
Affiliations: \\ ${ }^{1}$ Department of Respiratory Medicine, Research School NUTRIM, Maastricht University Medical Centre, \\ Maastricht, The Netherlands. \\ ${ }^{2}$ Department of Research and Education, $\mathrm{CIRO}+$, centre of expertise for chronic organ failure, Horn, The \\ Netherlands.
}

\section{Correspondence:}

Coby van de Bool, NUTRIM School of Nutrition and Translational Research in Metabolism, Department of Respiratory Medicine, Maastricht University Medical Centre+, PO Box 616, 6200 MD, Maastricht, The Netherlands. E-mail: coby.vandebooldmaastrichtuniversity.nl

ABSTRACT Decreased physical performance due to loss of muscle mass (i.e. sarcopenia) is prevalent in ageing and appears more pronounced in chronic disease. A comprehensive profile of the sarcopenic phenotype in chronic obstructive pulmonary disease (COPD) is not yet available. The aim of the present study was to characterise prevalence, functional implications and predictive value of sarcopenia with or without abdominal obesity in Dutch COPD patients eligible for pulmonary rehabilitation.

505 COPD patients (aged 37-87 years; 57\% male) underwent assessment of lung function, body composition and physical functioning, before entering pulmonary rehabilitation. Sarcopenia was assessed by appendicular skeletal muscle index (ASMI) and abdominal obesity by android/gynoid percentage fat mass $(\mathrm{A} / \mathrm{G} \% \mathrm{FM})$ using dual energy X-ray absorptiometry.

$86.5 \%$ of patients were sarcopenic and showed lower physical functioning, while coexistent abdominal obesity (78.0\%) resulted in higher physical functioning. Implications on endurance were less pronounced in women. The predictive value for physical functioning was higher for the "three-compartment" model (ASMI, bone mineral content and A/G\%FM) than the "two-compartment" model (fat-free mass index and fat mass index) or "one-compartment" model (body mass index).

In patients eligible for pulmonary rehabilitation, sarcopenia is highly prevalent in all body mass index categories and associated with impaired strength, and in men also with decreased endurance. Abdominal obesity seems to have protective effects on physical functioning. ASMI is a better predictor for physical functioning than fat-free mass index.

@ERSpublications

Highly prevalent sarcopenia and abdominal obesity in COPD patients exert antagonistic effects on physical functioning http://ow.ly/L5b9r 


\section{Introduction}

Chronic obstructive pulmonary disease (COPD) is a major public health problem, which is projected to rank third worldwide in terms of mortality in 2020 [1]. Evidence is accumulating that disease burden in COPD is largely determined by extra-pulmonary impairment, including skeletal muscle dysfunction, osteoporosis and cardiovascular disease [2]. Nutritional status is an important determinant of these extra-pulmonary effects. Today, it is generally accepted that body mass index (BMI) is limited to indicate differences in nutritional status. Indeed, we and others have previously shown hidden obesity in normal weight patients with COPD due to proportionally low muscle mass [3-5]. To characterise low muscle mass in COPD, traditionally a fat-free mass index (FFMI) below age- and sex-adjusted 10th-percentile values for healthy subjects is commonly used, corresponding to a FFMI $<17 \mathrm{~kg} \cdot \mathrm{m}^{-2}$ for males and $<15 \mathrm{~kg} \cdot \mathrm{m}^{-2}$ for females in normal to underweight Caucasian COPD patients [6]. This criterion was mainly developed to diagnose patients with or at risk for cachexia, a syndrome that causes generalised wasting of muscle mass [7]. It is expected that, in the current obesogenic society, the proportion of COPD patients with sarcopenic obesity will increase. Recently, FFMI percentiles were proposed that also adjust for BMI [8], but dual energy $\mathrm{X}$-ray absorptiometry (DEXA) nowadays also allows additional assessment of fat mass (FM) and fat-free mass at regional level besides assessment of bone mineral density. An appendicular skeletal muscle mass index (ASMI) of $\geqslant 2$ standard deviations below the mean of healthy persons between 20 and 30 years of age of the same ethnic group is nowadays commonly used to define sarcopenia in the elderly [9]. In the general well-functioning aged population, sarcopenia according to this definition is prevalent in $\sim 15 \%$ [10], but only sparse data are available in COPD. In a non-institutionalised civilian population of South Korea, sarcopenia was found in $32.8 \%$ of male and $12.2 \%$ of female COPD patients [11]. However, no data are yet available from a European population, or from patients eligible for pulmonary rehabilitation which are characterised by impaired physical functioning. Moreover, we recently demonstrated a high prevalence of abdominal obesity in patients eligible for pulmonary rehabilitation [12], but so far no data are available regarding the relative contribution of sarcopenia and abdominal obesity to impaired physical functioning in COPD. Therefore, the aim of the current study was to provide a comprehensive profile of the sarcopenic (low ASMI) with or without abdominal obesity (high android/gynoid percentage FM ratio (A/G\%FM)) phenotype in a representative group of Dutch COPD patients screened for pulmonary rehabilitation, by: 1) exploring its prevalence across all BMI categories; 2) investigating its functional implications; and 3) determining the predictive value of ASMI over BMI and FFMI for physical functioning. Because it is suggested that the relationship between body composition and functional capacity differs between males and females [13], we also explored putative sex differences. We hypothesise that functional consequences of low muscle mass can better be predicted by appendicular skeletal muscle mass than whole body fat free mass as the latter also includes a large part of water-containing organs and non-muscle tissue.

\section{Subjects and methods \\ Subjects}

COPD patients were recruited from $\mathrm{CIRO}+$, Centre of Expertise for Organ Failure (Horn, the Netherlands) between 2010 and 2011 [14]. Diagnosis of COPD was confirmed according to the Global Initiative for Chronic Obstructive Lung Disease (GOLD) guidelines [1], after referral for pulmonary rehabilitation by chest physicians from several hospitals in the south-east of the Netherlands. Patients were clinically stable and those without DEXA measurements were excluded. In total, 505 patients were included in the analyses. This retrospective study uses de-identified and pre-existing data for analyses and is institutional review board exempted.

\section{Measurements}

Measurements were performed at $\mathrm{CIRO}+$ before entering pulmonary rehabilitation, as part of a 3-day baseline assessment [14]. Sex and age were recorded from demographics.

\section{Lung function}

In accordance with the latest GOLD guidelines [1], standardised equipment (Masterlab ${ }^{\oplus}$ Jaeger, Würzburg, Germany) was used to assess post-bronchodilator forced expiratory volume in $1 \mathrm{~s}$ (FEV1) and forced vital capacity (FVC). Diffusing capacity of the lung for carbon monoxide (DLCO) was assessed using the single-breath method. All obtained values are expressed as percentages of the predicted value, by comparison with age and sex-specific reference values [15].

\section{Body composition}

Total body height was measured to the nearest $0.5 \mathrm{~cm}$ with a wall-mounted stadiometer and total body weight to the nearest $0.1 \mathrm{~kg}$ using a weighing scale. BMI was calculated as weight $/ \mathrm{height}\left(\mathrm{kg} \cdot \mathrm{m}^{-2}\right)$ and categorised according to the recent European Respiratory Society (ERS) statement on nutritional 
assessment and therapy in COPD [6]: BMI $<20 \mathrm{~kg} \cdot \mathrm{m}^{-2}$ (underweight); BMI 20-25 $\mathrm{kg} \cdot \mathrm{m}^{-2}$ (normal weight), BMI $25-30 \mathrm{~kg} \cdot \mathrm{m}^{-2}$ (overweight) and BMI $>30 \mathrm{~kg} \cdot \mathrm{m}^{-2}$ (obese). Body composition was measured using DEXA (Lunar Prodigy system; GE Healthcare, Madison, WI, USA). FFMI was calculated by dividing fat free mass (lean mass plus bone mineral content (BMC)) by height ${ }^{2}$, and FM by subtracting fat free mass from total weight. Low FFMI was defined as a FFMI $<17 \mathrm{~kg} \cdot \mathrm{m}^{-2}$ for men and $<15 \mathrm{~kg} \cdot \mathrm{m}^{-2}$ for women [6]. The ratio of the percentage FM in the android region (waist) to the percentage FM in the gynoid region (hip) was used as a measure for abdominal FM. Sarcopenia was defined according to cut-offs for ASMI ( $<7.23 \mathrm{~kg} \cdot \mathrm{m}^{-2}$ for men; $<5.67 \mathrm{~kg} \cdot \mathrm{m}^{-2}$ for women) [9]. An A/G\%FM $>1.0$ for men and $>0.8$ for women was applied to diagnose abdominal obesity [16]. Osteopenia was defined as a $\mathrm{T}$ score between -1 and -2.5 , osteoporosis as a T-score $\leqslant-2.5$ [17].

\section{Physical functioning}

A supervised symptom-limited cardiopulmonary incremental cycle test (CPET) was conducted on an electronically braked cycle ergometer (Carefusion, Houten, the Netherlands) to determine peak workload. Additionally, a constant work rate cycling endurance test (CWRT) was performed at $75 \%$ of the peak workload to determine cycle endurance time (CET). The best of two 6-min walk distance (6MWD) tests was selected for analyses. Peak quadriceps muscle strength was assessed during volitional isokinetic contractions (angular velocity of $90^{\circ}$ per second) on a Biodex (Biodex System 4 Pro; Biodex Medical Systems, Inc., New York, USA).

\section{C-reactive protein measurement}

Plasma levels of C-reactive protein (CRP) were assessed by high-sensitivity particle-enhanced immunoassay (COBAS Mira ${ }^{\oplus}$, Radiometer, Copenhagen, Denmark) and were used as a marker for systemic inflammation.

\section{Statistical analysis}

All statistical analyses were performed using the Statistical Package for Social Sciences version 20.0 (SPSS, Inc., Chicago, IL, USA). Two-sided p-values $<0.05$ were considered statistically significant. The ShapiroWilk test was used to assess data for normal distribution. Continuous variables were presented as median (interquartile range) and were compared between two groups by a t-test for independent samples (parametric data) or Mann-Whitney U-test (nonparametric data), and compared between more than two groups by one-way ANOVA (parametric data) or Kruskal-Wallis test (nonparametric data). Discrete variables were presented as percentages and compared using the Chi-squared test. Multivariate backward linear regression analyses were performed to determine the predictive values of body composition models to physical functioning outcomes.

\section{Results}

\section{Subject characteristics}

In general, the median age of the $505 \mathrm{COPD}$ patients was 64 years, $57 \%$ were male and the majority of them were former smokers (71.2\%), 26.2\% were current smokers, while $2.6 \%$ never smoked. COPD severity ranged from mild to very severe (GOLD I/II/III/IV: 7.9/40.8/39.8/11.5\%). Men were slightly older than women ( 66 versus 60 years, $\mathrm{p}<0.001$ ).

\section{Prevalence of body composition phenotypes}

$37.8 \%$ of patients had normal body weight, followed by $28.7 \%$ overweight, $18.8 \%$ obese and $14.7 \%$ underweight. Applying the ASMI cut-offs revealed sarcopenia in $83.0 \%$ of men and $91.2 \%$ of women, resulting in a total of $86.5 \%$. Almost $34 \%$ had a low FFMI. Further classification revealed abdominal obesity in the majority of the patients $(80.6 \%)$, as well as $78.0 \%$ in the sarcopenic patients.

Figure 1 shows that a highly prevalent sarcopenic phenotype across all BMI categories: 100\% in underweight, $96.9 \%$ in normal weight, $87.6 \%$ in overweight and $53.7 \%$ in obese patients. These proportions were comparable for both sexes, except in those overweight $(82.0 \%$ in men versus $96.4 \%$ in women, $\mathrm{p}<0.05)$. In contrast, the prevalence of a low FFMI rapidly reduced from $83.8 \%$ in underweight patients to $48.2 \%$ in normal weight patients and $13.8 \%$ in overweight patients but none of the obese patients (table 1). Coexistence of abdominal obesity and sarcopenia across BMI categories was comparable between sarcopenic men and women: $33.8 \%$ in underweight, $78.4 \%$ in normal weight, $94.5 \%$ in overweight and $100 \%$ in obese. Additionally, $97.1 \%$ of non-sarcopenic patients were abdominal obese.

In general, sarcopenic patients without abdominal obesity were younger and had lower diffusion capacity and CRP levels than sarcopenic patients with abdominal obesity (table 2). Patients with this combined phenotype showed more impaired lung function than non-sarcopenic patients. Per definition, additional differences in body composition were found across body composition phenotypes regarding BMI, FFMI, 


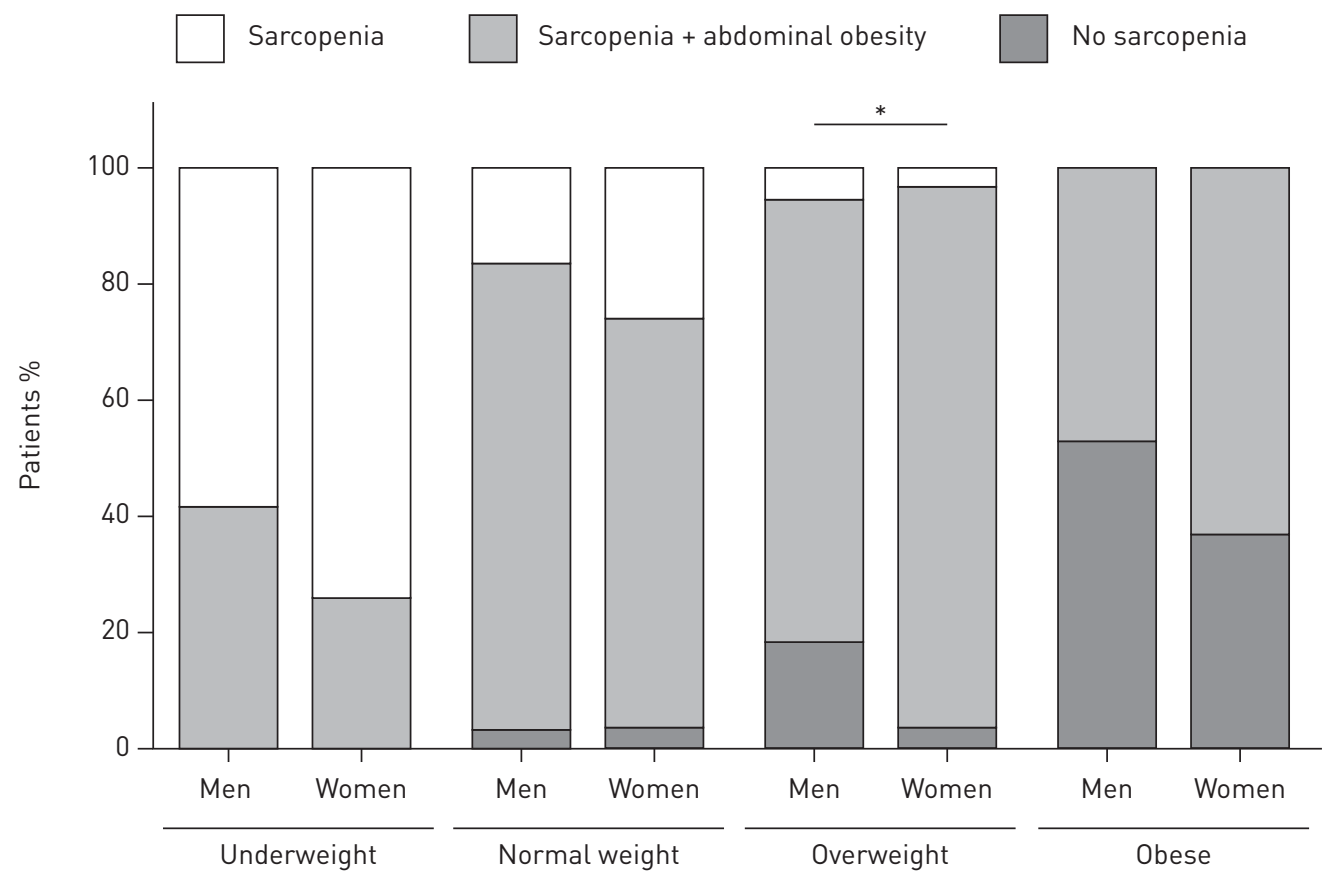

FIGURE 1 Prevalence of sarcopenia, in presence and absence of abdominal obesity, across body mass index categories. $*$ : $\mathrm{p}<0.05$.

fat mass index (FMI), fat percentage and android FM. Prevalence of osteoporosis was highest in the sarcopenic patients without abdominal obesity.

Functional implications

Sarcopenic patients without abdominal obesity performed less during the different tests than non-sarcopenic patients, reflected by lower muscle strength and peak workload, as well as a shorter CET and 6MWD in men

\begin{tabular}{|c|c|c|c|}
\hline & Low FFMI & Normal FFMI & Total \\
\hline \multicolumn{4}{|l|}{ Underweight } \\
\hline Non-sarcopenic & $0(0.0)$ & $0(0.0)$ & $0(0.0)$ \\
\hline Sarcopenic & $62(83.8)$ & $12(16.2)$ & $74(100.0)$ \\
\hline Total & 62 (83.8) & $12(16.2)$ & $74(100.0)$ \\
\hline \multicolumn{4}{|l|}{ Normal weight } \\
\hline Non-sarcopenic & $0(0.0)$ & $6(3.1)$ & $6(3.1)$ \\
\hline Sarcopenic & 92 (48.2) & 93 (48.7) & 185 (96.9) \\
\hline Total & $92(48.2)$ & 99 (51.8) & $191(100.0)$ \\
\hline \multicolumn{4}{|l|}{ Overweight } \\
\hline Non-sarcopenic & $0(0.0)$ & $18(12.4)$ & $18(12.4)$ \\
\hline Sarcopenic & 20 (13.8) & 107 (73.8) & $127(87.6)$ \\
\hline Total & 20 (13.8) & 125 (86.2) & $145(100.0)$ \\
\hline \multicolumn{4}{|l|}{ Obese } \\
\hline Non-sarcopenic & $0(0.0)$ & $44(46.3)$ & $44(46.3)$ \\
\hline Sarcopenic & $0(0.0)$ & 51 (53.7) & 51 (53.7) \\
\hline Total & $0(0.0)$ & $95(100.0)$ & $95(100.0)$ \\
\hline \multicolumn{4}{|l|}{ Total } \\
\hline Non-sarcopenic & $0(0.0)$ & 68 (13.5) & $68(13.5)$ \\
\hline Sarcopenic & 174 (34.5) & $263(52.1)$ & 437 (86.5) \\
\hline Total & 174 (34.5) & 331 (65.5) & 505 (100.0) \\
\hline
\end{tabular}

Data are presented as $\mathrm{n}(\%)$. 
TABLE 2 Subjects characteristics per body composition phenotype

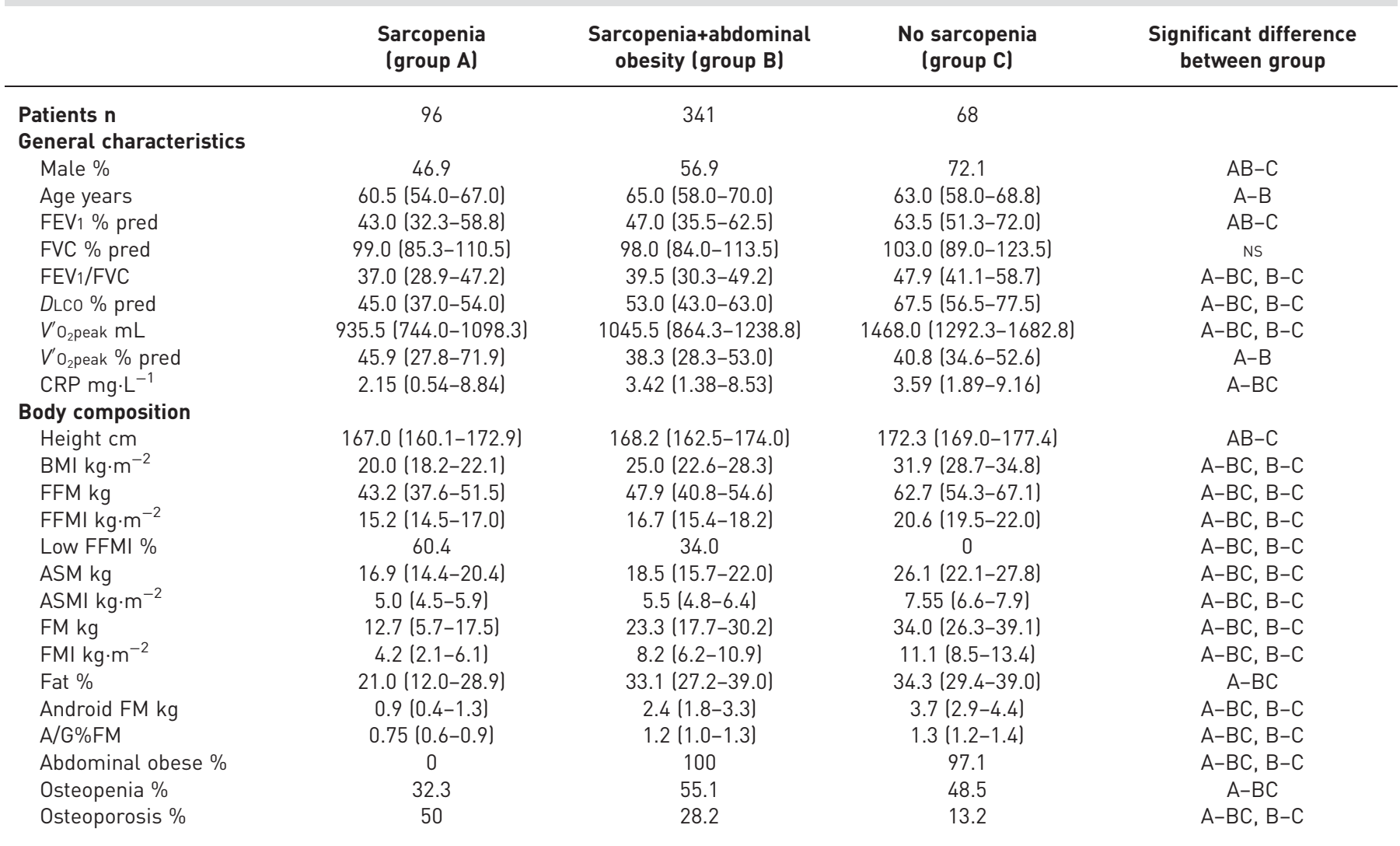

Data are presented as median (interquartile range), unless otherwise stated. FEV1: forced expiratory volume in $1 \mathrm{~s} ; \%$ pred: \% predicted; FVC: forced vital capacity; DLCo: diffusing capacity of the lung for carbon monoxide; $V^{\prime} 0_{2}$ peak: peak oxygen uptake; CRP: C-reactive protein; BMI: body mass index; FFM: fat-free mass; FFMI: fat-free mass index; ASM: appendicular skeletal muscle; ASMI: appendicular skeletal muscle index; FM: fat mass; FMI: fat mass index; A/G\%FM: android/gynoid percentage fat mass; Ns: nonsignificant.

(fig. 2). In case of concomitant abdominal obesity, sarcopenic patients achieved higher muscle strength. Moreover, sarcopenic men with abdominal obesity achieved higher peak workload and longer CET. Nonetheless, physical functioning was still significantly lower compared with non-sarcopenic patients, including muscle strength, peak workload, CET and 6MWD (CET and 6MWD only in men).

To assess the ASM quality between body composition phenotypes, functional measures were also compared expressed per kilogram ASM (table 3). Both in men and women, muscle strength expressed per kilogram ASM was lower in those who were sarcopenic without abdominal obesity compared to those who were sarcopenic with abdominal obesity. In men only, muscle strength was highest in the non-sarcopenic patients compared to sarcopenic patients with or without abdominal obesity. Furthermore, again in men, peak workload and peak oxygen uptake $\left(V^{\prime} \mathrm{O}_{2}\right.$ peak $)$ per $\mathrm{kg}$ ASM was lower in both sarcopenic groups compared with the non-sarcopenic group, while the CET per kg ASM was lowest in the sarcopenic group without abdominal obesity compared to the other two groups.

\section{Predictive value}

Table 4 shows the results of the sex-specific backward-regression analyses on the predictive value of "one-compartment" (BMI), "two-compartment" (FFMI+FMI) and "three-compartment" (ASMI+BMC+A/ $\mathrm{G} \% \mathrm{FM}$ ) assessment of body composition, corrected for age and FEV1. BMI was a significant predictor for muscle strength, peak workload and 6MWD (6MWD only in women). The one-compartment model explained less than the two-compartment model, which explained 30-31\% of total muscle strength variability, with FFMI and FMI as positive predictors (FMI only in women). The predictive value increased even more to $47-51 \%$ in the three-compartment model, in which ASMI, BMC and A/G\%FM retained positive predictors. At the level of peak workload, FFMI retained a positive predictor in the two-compartment model, explaining $41-48 \%$ of total variability, while $47-56 \%$ was explained by the three-compartment model, with positive roles for ASMI and BMC (BMC only in men). Regarding CET, 


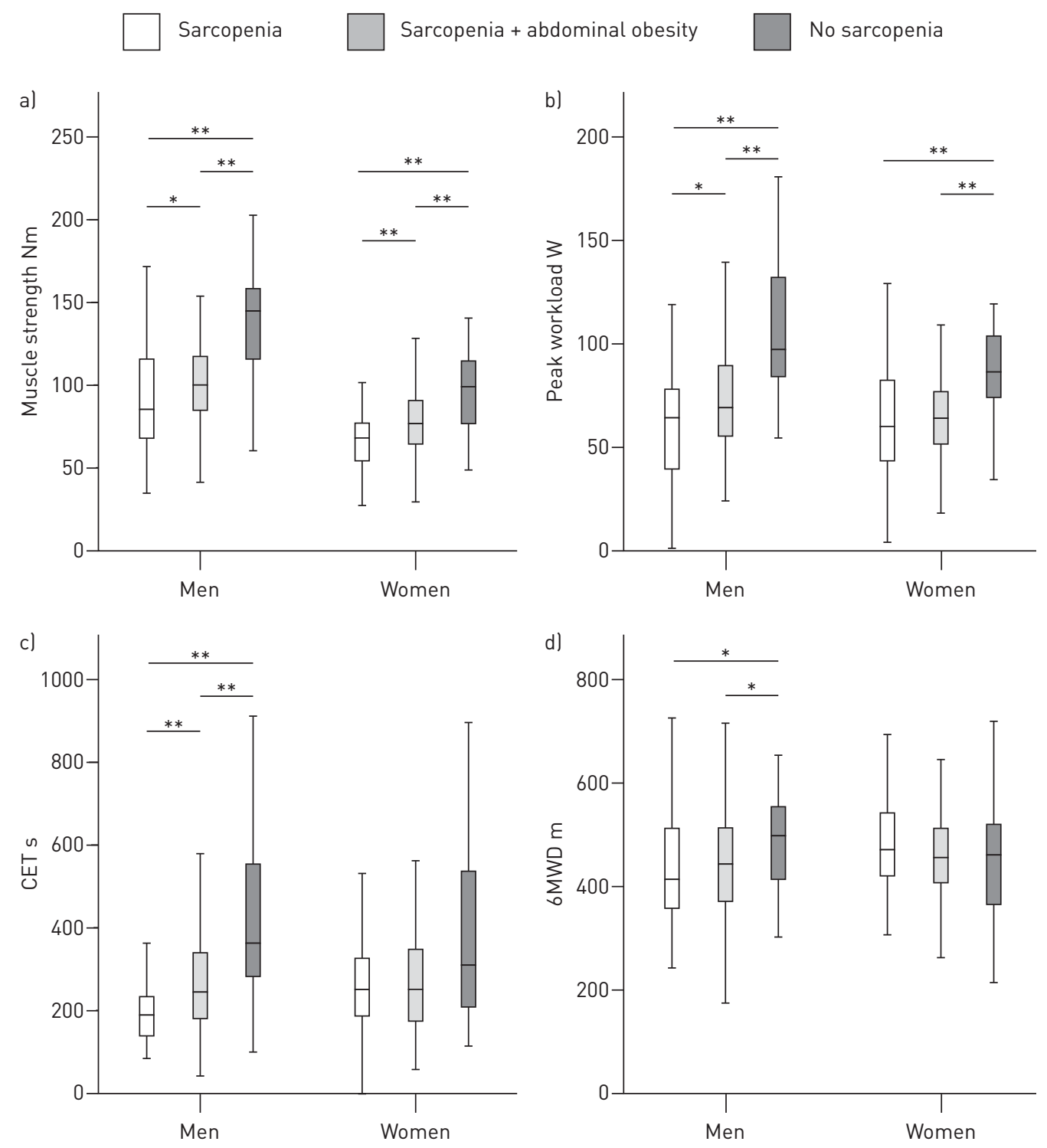

FIGURE 2 Functional implications of sarcopenia in presence or absence of abdominal obesity. CET: cycle endurance time; 6MWD: 6-min walk distance. ${ }^{*}: \mathrm{p}<0.05 ;{ }^{*}$ : $\mathrm{p}<0.01$.

TABLE 3 Qualitative implications of sarcopenia (with or without abdominal obesity)

\section{Sarcopenia \\ (group A)}

\section{Patients $n$}

Men

Muscle strength/ASM Nm.kg ${ }^{-1}$

Peak workload/ASM W. $\mathrm{kg}^{-1}$

$V^{\prime} \mathrm{O}_{2}$ peak/ASM mL$\cdot \mathrm{kg}^{-1}$

CET/ASM s $\cdot \mathrm{kg}^{-1}$

\section{Women}

Muscle strength/ASM Nm $\mathrm{kg}^{-1}$

Peak workload/ASM W. $\mathrm{kg}^{-1}$

$V^{\prime} \mathrm{O}_{2}$ peak/ASM $\mathrm{mL} \cdot \mathrm{kg}^{-1}$

CET/ASM s. $\mathrm{kg}^{-1}$
96

$4.2(3.4-5.4)$
$3.2(2.3-3.8)$
$47.6(40.2-53.8)$
$9.9(7.2-12.1)$

$4.5(3.9-5.1)$

$4.0(3.1-5.3)$

$60.3(51.7-70.7)$

$17.8(12.4-23.2)$

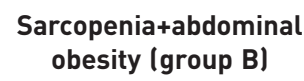

341
No sarcopenia (group C)

68

$5.2(4.3-6.0)$
$3.6(3.2-4.6)$
$55.0(49.3-60.7)$
$13.5(10.6-20.8)$

$$
\begin{gathered}
4.7(3.7-5.7) \\
4.4(3.8-4.9) \\
73.1(57.2-74.9) \\
14.1(10.1-27.2)
\end{gathered}
$$

Significant difference between group

Data are presented as median (interquartile range), unless otherwise stated. Comparison of significant physical functioning measures expressed per kilogram appendicular skeletal muscle (ASM) across body composition phenotypes. $V^{\prime} O_{2}$ peak: peak oxygen uptake; $C E T$ : cycle endurance time; NS: nonsignificant. 
TABLE 4 Predictive value of body composition assessment models

\begin{tabular}{|c|c|c|c|c|c|c|}
\hline \multirow[t]{2}{*}{ Predictors } & \multicolumn{3}{|c|}{ Men } & \multicolumn{3}{|c|}{ Women } \\
\hline & Adjusted $\mathrm{R}^{2}$ & $\beta$ & $p$-value & Adjusted $\mathbf{R}^{2}$ & $\beta$ & p-value \\
\hline \multicolumn{7}{|c|}{ Muscle strength } \\
\hline \multicolumn{7}{|c|}{ One-compartment model } \\
\hline BMI & 0.29 & 2.50 & 0.00 & 0.30 & 1.58 & 0.00 \\
\hline \multicolumn{7}{|c|}{ Two-compartment model } \\
\hline FFMI & 0.32 & 6.28 & 0.00 & 0.31 & 3.41 & 0.00 \\
\hline FMI & & & NS & & 0.97 & 0.03 \\
\hline \multicolumn{7}{|c|}{ Three-compartment model } \\
\hline ASMI & 0.47 & 17.01 & 0.00 & 0.51 & 11.74 & 0.00 \\
\hline $\mathrm{BMC}$ & & 0.01 & 0.00 & & 0.02 & 0.00 \\
\hline $\mathrm{A} / \mathrm{G} \% \mathrm{FM}$ & & & NS & & 16.68 & 0.00 \\
\hline \multicolumn{7}{|c|}{ Peak workload } \\
\hline \multicolumn{7}{|c|}{ One-compartment model } \\
\hline BMI & 0.47 & 0.96 & 0.00 & 0.40 & 0.56 & 0.02 \\
\hline \multicolumn{7}{|c|}{ Two-compartment model } \\
\hline FFMI & 0.48 & 2.90 & 0.00 & 0.41 & 1.92 & 0.01 \\
\hline FMI & & & NS & & & NS \\
\hline \multicolumn{7}{|c|}{ Three-compartment model } \\
\hline ASMI & 0.56 & 8.06 & 0.00 & 0.47 & 10.68 & 0.00 \\
\hline $\mathrm{BMC}$ & & 0.01 & 0.00 & & & NS \\
\hline $\mathrm{A} / \mathrm{G} \% \mathrm{FM}$ & & & NS & & & NS \\
\hline \multicolumn{7}{|l|}{ CET } \\
\hline \multicolumn{7}{|c|}{ One-compartment model } \\
\hline BMI & 0.02 & & NS & 0.02 & & NS \\
\hline \multicolumn{7}{|c|}{ Two-compartment model } \\
\hline FFMI & 0.02 & 17.53 & 0.01 & 0.02 & & NS \\
\hline & & & & & & NS \\
\hline \multicolumn{7}{|c|}{ Three-compartment model } \\
\hline ASMI & 0.07 & 42.31 & 0.03 & 0.04 & & NS \\
\hline $\mathrm{BMC}$ & & 0.08 & 0.03 & & 0.10 & 0.02 \\
\hline $\mathrm{A} / \mathrm{G} \% \mathrm{FM}$ & & & NS & & & NS \\
\hline \multicolumn{7}{|l|}{ 6MWD } \\
\hline \multicolumn{7}{|c|}{ One-compartment model } \\
\hline BMI & 0.14 & & NS & 0.26 & -5.47 & 0.00 \\
\hline \multicolumn{7}{|c|}{ Two-compartment model } \\
\hline FFMI & 0.16 & 7.19 & 0.04 & 0.27 & & NS \\
\hline FMI & & -5.43 & 0.01 & & -7.62 & 0.00 \\
\hline \multicolumn{7}{|c|}{ Three-compartment model } \\
\hline ASMI & 0.16 & & NS & 0.20 & & NS \\
\hline $\mathrm{BMC}$ & & & NS & & & NS \\
\hline $\mathrm{A} / \mathrm{G} \% \mathrm{FM}$ & & 64.52 & 0.01 & & -60.45 & 0.04 \\
\hline
\end{tabular}

Summary of backward multiple regression analysis, with muscle strength, peak workload, cycling endurance time (CET) and 6-min walk distance (6MWD) as dependent variables and body composition models (one-compartment: body mass index (BMI); two-compartment: fat-free mass index (FFMI) and fat mass index (FMI); or three-compartment: appendicular skeletal muscle index (ASMI), bone mineral content (BMC) and android/gynoid percentage fat mass (A/G\%FM)) as predictors. All models are adjusted for age and forced expiratory volume in $1 \mathrm{~s}$. NS: nonsignificant.

$2 \%$ of variability was explained by the two-compartment model, with FFMI as positive predictor (only in men), whereas 4-7\% was explained by the model, including ASMI and BMC as positive predictors (ASMI only in men). Furthermore, the two-compartment model explained $16-27 \%$ of total variability in $6 \mathrm{MWD}$, in which FFMI retained a positive predictor in men and FMI a negative predictor in men and women. Explained variability decreased to $16-20 \%$ by the three-compartment model, comprising A/G\%FM as a positive predictor in men, but a negative predictor for 6MWD in women.

\section{Discussion}

This is the first study presenting the prevalence of sarcopenia and abdominal obesity, to characterise its functional implications and predictive value for physical functioning in a representative group of Dutch COPD patients eligible for pulmonary rehabilitation. 
To date, the prevalence of sarcopenia has only been explored in a non-institutionalised civilian population of South Korea (32.8\% of male and $12.2 \%$ of female COPD patients) [11]. Clearly, the current proportions overshadow the prevalence in South Korea, but the latter was reported in a relatively healthy population, as individuals admitted to hospital or nursing homes were not included. The higher prevalence of sarcopenia in COPD than in the healthy elderly (based on available literature [10]) reflects the suggested accelerated ageing in COPD, which is further supported by the reported shorter telomere length in COPD patients [18]. Potential catabolic triggers for inducing the sarcopenic process in COPD include physical inactivity, oxidative stress, inflammation, use of glucocorticosteroids and hypoxia [19]. As described in the recently published ERS statement on nutritional assessment and therapy in COPD [6], abnormally low FFMI in normal-to-underweight COPD patients is based on well-established adverse effects of low FFMI on physical performance and survival, and defined as a FFMI below age- and sex-specific 10th-percentile values. Depending on the cut-off value, prevalence rates range from 15 to 35 [3,20,21], but are low or absent in overweight and obese subjects. Although we did not use BMI-specific ASMI reference values as recently proposed by PRADO et al. [22], a high prevalence of the sarcopenic phenotype persisted across all BMI categories, including $74.5 \%$ of overweight and $53.7 \%$ of obese patients. The ASMI cutoffs for sarcopenia are therefore particularly useful to diagnose abnormal body composition in overweight and obese patients. This concept has also been observed in patients with non-small cell lung cancer, in which $59 \%$ of the overweight patients were sarcopenic [23].

For the diagnosis of sarcopenia, the European Working Group on Sarcopenia in Older People (EWGSOP) recommends using the presence of low physical function (strength or performance) next to low ASMI [9]. One study aimed to verify the association between ASMI and physical functioning in older COPD outpatients [24]. However, they only assessed 6MWD as a measure for physical functioning and concluded that FEV1 was a stronger predictor of physical functioning in COPD patients than body composition. Another study demonstrated that skeletal muscle weakness in COPD patients was due to loss of ASM, but was not related to airflow obstruction [25]. We explored the relation between low ASMI and a comprehensive set of functional outcome measures in the current population of COPD patients, as the relative influence of muscle mass versus impaired lung function on performance might differ between tests $[24,26,27]$. Indeed, we showed that sarcopenia (in general and expressed per kilogram ASM) is not only accompanied by impaired muscle strength, but also with impaired exercise performance, which was more pronounced for peak workload than for 6MWD. Although actual $V^{\prime} \mathrm{O}_{2}$ peak was lowest in sarcopenic patients, they showed the highest $V^{\prime} \mathrm{O}_{2}$ peak expressed as \% predicted. This is due to the lower predicted values for this group, which are based on weight-class. Non-sarcopenic patients were generally overweight to obese and overall showed better physical functioning, typically reflecting the "obesity paradox" (stating that obesity is, on one hand, associated with better survival and some functional outcomes but, on the other hand, also associated with increased risk of cardiovascular and metabolic diseases). Referring to the high prevalence of abdominal obesity in COPD patients [12] and, moreover, in sarcopenic patients [11], it was relevant to investigate if and in what direction coexistence of abdominal obesity further affected physical functioning in COPD patients. This group of sarcopenic patients with concomitant abdominal obesity generally displayed a higher physical functioning than sarcopenic patients without abdominal obesity. This finding corresponds to previous literature reporting that exercise capacity is preserved or even increased in obese COPD patients during cycling (non-weight bearing exercise) [28, 29], except when walking is used as testing modality (weight bearing exercise) [30]. A potential explanation for this functional improvement in advanced COPD might be a lowering effect of obesity on resting and dynamic hyperinflation as established determinant of exercise impairment in COPD [29, 31]. Nonetheless, this condition of excessive body weight with a disproportional low muscle mass (i.e. sarcopenic obesity) may exert cumulative risks on metabolic and cardiovascular health, resulting in increased morbidity and mortality [32]. Loss of muscle mass can result in decreased muscle oxidative capacity which is related to metabolic disorders including diabetes and metabolic syndrome in non-obese individuals [33]. Decreased oxidative capacity may even be more pronounced in COPD due to a muscle fibre I to II shift. Indeed, a recent population study demonstrated an association between sarcopenia and metabolic syndrome in male COPD patients [11]. Although conclusive data were not available in the present study, abdominal obesity was accompanied by higher CRP-levels, corresponding with recent data indicating that excessive abdominal FM contributes to the systemic inflammation in COPD, which in turn is an important risk factor for cardiovascular disease [4, 34]. Albeit abdominal obesity seem to be protective in the present study, the effect on cardiovascular co-morbidity should be further evaluated in subsequent study in order to be able to point out the net effect of this phenotype.

In our COPD patients, a three-compartment body composition model (including ASMI, BMC and A/G\% FM) had a higher predictive value for physical functioning than a two-compartment model (including FFMI and FMI) or one-compartment model (BMI). Nonetheless, differences were found in the predictive values of the body composition variables when comparing physical functioning outcomes, but also when 
comparing men with women. ASMI positively contributed to muscle strength, peak workload and CET (CET only in men), whereas A/G\%FM positively contributed to muscle strength and 6MWD in men, but negatively to 6MWD in women. The better prediction of ASMI for muscle strength and peak workload as compared with CET and 6MWD can be attributed to the direct interaction between muscle mass and weakness in this population, whereas muscle endurance is suggested to be affected by multiple additional factors related to COPD [35]. Noticeable is the independent contribution of BMC to endurance in the regression model. Lower functional performance has been described in patients with osteoporosis, but a contribution of the bone mass to functional performance has never been shown so far.

Our analyses revealed more sex variations, as all reported physical functioning outcomes significantly differed across body composition phenotypes in men, but in women no differences could be detected in CET and 6MWD across body composition phenotypes. Moreover, the coexistence of abdominal obesity next to sarcopenia resulted in higher muscle strength, peak workload and CET in men, while this only applied for muscle strength in women. Therefore, especially sarcopenic men might be protected for physical functioning by the presence of abdominal obesity. However, the reason for the sex difference in the functional impact of abdominal obesity is unclear. Nonetheless, sex differences in the degree of muscle function impairment have also been found in cancer patients [36, 37]. Its underlying mechanism may differ between men and women, suggesting that the degree of physical impairment is affected by sexual dimorphism [36].

Some shortcomings of the current study need to be considered. Because of the cross-sectional nature of our data, we were not able to identify cause-effect relationships. Another limitation was the lack of data on (unintended) weight loss to assess the relative contribution of cachexia (unintentional weight loss $>5 \%$ in 6 months and low FFMI) to sarcopenia. Additionally, the current results cannot be generalised to the whole COPD population, because the studied patient sample consisted exclusively of patients referred for pulmonary rehabilitation. However, these patients pertain an interesting population because of their functional impairments.

Future research is necessary to further unravel the causes and effects of sarcopenia and abdominal obesity in COPD. Nonetheless, the current findings indicate that assessment of the three-compartimental body composition (ASMI, A/G\%FM and BMC) might be favourable over distinction between FFMI and FMI, indicated by 1) the high persisting prevalence's of sarcopenia and abdominal obesity in all BMI categories; 2) its discrimination for impaired muscle strength and endurance; and 3) the higher predictive value of the three-compartment regression model compared to the one- or two-compartment models.

\section{Conclusion}

This paper shows that sarcopenia is highly prevalent across all BMI categories in patients eligible for pulmonary rehabilitation in the Netherlands. Moreover, we showed loss of muscle strength in sarcopenic patients and in men also impaired endurance. The coexistence of abdominal obesity might protect against impaired physical functioning in sarcopenic patients. The predictive value for physical functioning was higher for the "three-compartment"-model (ASMI, BMC and A/G\%FM) than the "two-compartment"-model (FFMI and FMI) or "one-compartment"-model (BMI). The mechanisms underlying these observations remain to be elucidated.

\section{Acknowledgements}

Each author has participated sufficiently, intellectually or practically, in the work to take public responsibility for the content of the article, including the conception, design and conduct of the experiment and for data interpretation. The contribution of the authors to the manuscript is as follows: CvdB: study design, analyzing data, drafting the manuscript, primary responsibility of the final content; ER: study design, reviewing the manuscript; FF and EW: reviewing the manuscript; AS: study design, drafting the manuscript and primary responsibility of the final content, reviewing the manuscript.

\section{References}

1 Vestbo J, Hurd SS, Agustí AG, et al. Global Strategy for the Diagnosis, Management, and Prevention of Chronic Obstructive Pulmonary Disease: GOLD Executive Summary. Am J Respir Crit Care Med 2013; 187: 347-365.

2 Patel AR, Hurst JR. Extrapulmonary comorbidities in chronic obstructive pulmonary disease: state of the art. Expert Rev Respir Med 2011; 5: 647-662.

3 Schols AM, Soeters PB, Dingemans AM, et al. Prevalence and characteristics of nutritional depletion in patients with stable COPD eligible for pulmonary rehabilitation. Am Rev Respir Dis 1993; 147: 1151-1156.

4 Rutten EP, Breyer MK, Spruit MA, et al. Abdominal fat mass contributes to the systemic inflammation in chronic obstructive pulmonary disease. Clin Nutr 2010; 29: 756-760.

5 Marquis K, Maltais F, Duguay V, et al. The metabolic syndrome in patients with chronic obstructive pulmonary disease. J Cardiopulm Rehabil 2005; 25: 226-232.

6 Schols AM, et al. Nutritional assessment and therapy in COPD: a European Respiratory Society statement. Eur Respir J 2014; 44: 1504-1520.

7 Engelen MP, Schols AM, Lamers RJ, et al. Different patterns of chronic tissue wasting among patients with chronic obstructive pulmonary disease. Clin Nutr 1999; 18: 275-280. 
8 Franssen FM, et al. New Reference Values for Body Composition by Bioelectrical Impedance Analysis in the General Population: Results From the UK Biobank. J Am Med Dir Assoc 2014; 15: 448.

9 Cruz-Jentoft AJ, Baeyens JP, Bauer JM, et al. Sarcopenia: European consensus on definition and diagnosis. Age Ageing 2010; 39: 412-423.

10 Cherin P, Voronska E, Fraoucene N, et al. Prevalence of sarcopenia among healthy ambulatory subjects: the sarcopenia begins from 45 years. Aging Clin Exp Res 2014; 26: 137-146.

11 Chung JH, Hwang $\mathrm{HJ}$, Han $\mathrm{CH}$, et al. Association between Sarcopenia and Metabolic Syndrome in Chronic Obstructive Pulmonary Disease: The Korea National Health and Nutrition Examination Survey (KNHANES) from 2008 to 2011. COPD 2015; 12: 82-89.

12 van de Bool C, Mattijssen-Verdonschot C, van Melick PP, et al. Quality of dietary intake in relation to body composition in patients with chronic obstructive pulmonary disease eligible for pulmonary rehabilitation. Eur $J$ Clin Nutr 2014; 68: 159-165.

13 Schols AM, Broekhuizen R, Weling-Scheepers CA, et al. Body composition and mortality in chronic obstructive pulmonary disease. Am J Clin Nutr 2005; 82: 53-59.

14 Spruit MA, Vanderhoven-Augustin I, Janssen PP, et al. Integration of pulmonary rehabilitation in COPD. Lancet 2008; 371: 12-13.

15 Clausen JL, Coates AL, Quanjer PH. Measurement of lung volumes in humans: review and recommendations from an ATS/ERS workshop. Eur Respir J 1997; 10: 1205-1206.

16 Bjorntorp, P. Regional patterns of fat distribution. Ann Intern Med 1985; 103: 994-995.

17 WHO Scientific Group on the Prevention and Management of Osteoporosis. Prevention and management of osteoporosis; report of a WHO scientific group. 2003. Available from http://whqlibdoc.who.int/trs/who_trs_921.pdf Date last accessed: April 1, 2015.

18 Houben JM, Mercken EM, Ketelslegers HB, et al. Telomere shortening in chronic obstructive pulmonary disease. Respir Med 2009; 103: 230-236.

19 Remels AH, Gosker HR, Langen RC, et al. The mechanisms of cachexia underlying muscle dysfunction in COPD. J Appl Physiol 2013; 114: 1253-1262.

20 Vestbo J, Prescott E, Almdal T, et al. Body mass, fat-free body mass, and prognosis in patients with chronic obstructive pulmonary disease from a random population sample: findings from the Copenhagen City Heart Study. Am J Respir Crit Care Med 2006; 173: 79-83.

21 Vermeeren MA, Creutzberg EC, Schols AM, et al. Prevalence of nutritional depletion in a large out-patient population of patients with COPD. Respir Med 2006; 100: 1349-1355.

22 Prado CM, Siervo M, Mire E, et al. A population-based approach to define body-composition phenotypes. Am Clin Nutr 2014; 99: 1369-1377.

23 Baracos VE, Reiman T, Mourtzakis M, et al. Body composition in patients with non-small cell lung cancer: a contemporary view of cancer cachexia with the use of computed tomography image analysis. Am J Clin Nutr 2010; 91: 1133S-1137S.

24 Cesari M, Pedone C, Chiurco D, et al. Physical performance, sarcopenia and respiratory function in older patients with chronic obstructive pulmonary disease. Age Ageing 2012; 41: 237-241.

25 Engelen MP, Schols AM, Does JD, et al. Skeletal muscle weakness is associated with wasting of extremity fat-free mass but not with airflow obstruction in patients with chronic obstructive pulmonary disease. Am J Clin Nutr 2000; 71: 733-738.

26 Gosselink R, Troosters T, Decramer M. Peripheral muscle weakness contributes to exercise limitation in COPD. Am J Respir Crit Care Med 1996; 153: 976-980.

27 Andrianopoulos V, Wagers SS, Groenen MT, et al. Characteristics and determinants of endurance cycle ergometry and six-minute walk distance in patients with COPD. BMC Pulm Med 2014; 14: 97.

28 Ora J, Laveneziana P, Ofir D, et al. Combined effects of obesity and chronic obstructive pulmonary disease on dyspnea and exercise tolerance. Am J Respir Crit Care Med 2009; 180: 964-971.

29 Ora J, Laveneziana $\mathrm{P}$, Wadell $\mathrm{K}$, et al. Effect of obesity on respiratory mechanics during rest and exercise in COPD. J Appl Physiol 2011; 111: 10-19.

30 Sava F, Laviolette L, Bernard S, et al. The impact of obesity on walking and cycling performance and response to pulmonary rehabilitation in COPD. BMC Pulm Med 2010; 10: 55.

31 Laviolette L, Sava F, O’Donnell DE, et al. Effect of obesity on constant workrate exercise in hyperinflated men with COPD. BMC Pulm Med 2010; 10: 33.

32 Biolo G, Cederholm T, Muscaritoli M. Muscle contractile and metabolic dysfunction is a common feature of sarcopenia of aging and chronic diseases: From sarcopenic obesity to cachexia. Clin Nutr 2014; 33: 737-748.

33 Moon SS. Low skeletal muscle mass is associated with insulin resistance, diabetes, and metabolic syndrome in the Korean population: the Korea National Health and Nutrition Examination Survey (KNHANES) 2009-2010. Endocr J 2014; 61: 61-70.

34 van den Borst B, Gosker HR, Koster A, et al. The influence of abdominal visceral fat on inflammatory pathways and mortality risk in obstructive lung disease. Am J Clin Nutr 2012; 96: 516-526.

35 Malaguti C, Napolis LM, Villaça D, et al. Relationship between peripheral muscle structure and function in patients with chronic obstructive pulmonary disease with different nutritional status. J Strength Cond Res 2011; 25: 1795-1803

36 Stephens NA, Gray C, MacDonald AJ, et al. Sexual dimorphism modulates the impact of cancer cachexia on lower limb muscle mass and function. Clin Nutr 2012; 31: 499-505.

37 Palomares MR, Sayre JW, Shekar KC, et al. Gender influence on weight-loss pattern and survival of nonsmall cell lung carcinoma patients. Cancer 1996; 78: 2119-2126. 